\title{
Modelamiento de la calidad del aire en la ciudad de Cuenca-Ecuador
}

\section{Modeling air quality in the city of Cuenca-Ecuador}

\author{
Diana Lucía Moscoso-Vanegas \\ Ph. D. (c). Ciencias Técnicas \\ Universidad de Cuenca \\ Cuenca, Ecuador \\ diana.moscoso@ucuenca.edu.ec
}

\author{
Verónica Eulalia Vázquez-Freire \\ Ingeniera Química \\ Universidad de Cuenca \\ Cuenca, Ecuador \\ vevazquezf@gmail.com
}

\author{
Ana Lucía Astudillo-Alemán \\ M. Sc. Toxicología Industrial y Ambiental \\ Universidad de Cuenca \\ Cuenca, Ecuador \\ anilua33@hotmail.com
}

\begin{abstract}
Resumen- El monitoreo de la calidad de aire en la ciudad de Cuenca detectó problemas de contaminación, iniciándose así un trabajo más profundo sobre la dispersión y transporte de los contaminantes descubiertos. Se trabajó con los modelos de dispersión DISPER5.2 y WRF/Chem, analizándose la calidad de modelación, grado de aproximación y limitantes. El Sistema de Información Geográfico ArcGIS permitió visualizar los mapas generados en los modelos. DISPER5.2 presentó una modelación de baja calidad debido a una limitada resolución en la simulación. La modelación es puntual, no hay relación entre todos los datos de entrada. WRF/ Chem estableció una relación entre los datos geográficos y meteorológicos de la zona de estudio con la química de los contaminantes, reportando resultados con una alta resolución, tanto en áreas locales como globales. Además, se evidenció la falta de información referente a emisiones de contaminantes para la alimentación del modelo y de equipos de monitoreo continuo.
\end{abstract}

Palabras clave- Calidad del aire, DISER5.2, WRF/ CHEM, ArcGIS.

Abstract- The air quality monitoring performed in the city of Cuenca, pollution problems became evident, therefore a more detailed study related to the dispersion and transport of contaminants was conducted. In this context two dispersion models such as: DISPER5.2 and WRF/Chem, were applied to analyze the quality of modeling, degree of approximation and limitations. The Geographic Information System ArcGIS allowed visualization the maps generated from the models. The DISPER5.2 model showed a low quality modeling, due to limited resolution simulation, modeling is punctual and no relationship between all input data. The second model (WRF/Chem), established a relationship between geographic and meteorological data of the study area with chemistry and pollutant dispersion, reporting re- sults with high resolution in both: local and global areas. In addition, the lack of information regarding emissions of pollutants to feed the model and continuous monitoring devices was evident.

Key words- Air Quality, DISER5.2, WRF/CHEM, ArcGIS.

\section{INTRODUCCIÓN}

En la actualidad, el estudio de los procesos que sufren los contaminantes emitidos a la atmósfera se basa en el uso de modelos numéricos que simulan los procesos físicos y químicos de dichos contaminantes. Estos modelos son ampliamente utilizados por los organismos encargados de controlar la contaminación del aire, para identificar las contribuciones de origen y ayudar en el diseño de estrategias efectivas de control de la contaminación. La United States Environmental Protection Agency (USEPA) [1] manifiesta que los modelos pueden ser utilizados para predecir las concentraciones futuras de contaminantes provenientes de múltiples fuentes después de la implementación de programas de regulación, con el fin de evaluar la efectividad de dichos programas.

Dentro de los modelos de dispersión existe normalmente un módulo químico que representa los procesos de transformación química y de fase, un módulo meteorológico que entrega los campos de vientos, temperatura, humedad, etc., que son necesarios para la resolución de la ecuación de 
continuidad de cada contaminante y un módulo que resuelve los procesos de transporte como la advección, convección, turbulencia, entre otros; todos estos módulos están conectados con bases de datos como son los inventarios de emisiones, datos de topografía y usos de suelos de la zona [2].

Existen varios tipos de modelos matemáticos para estudiar la dispersión de los contaminantes atmosféricos. Los modelos gaussianos son de uso común para el estudio de fuentes puntuales como las chimeneas industriales, mientras que los de escala local y regional son herramientas de gestión de calidad del aire ya que consideran los efectos de múltiples fuentes, contaminantes primarios y secundarios, procesos de deposición y meteorología local y regional. Los modelos globales al igual que los locales y regionales abarcan múltiples variables y, además, resuelven las ecuaciones de continuidad para toda la atmósfera [3].

Entre los modelos gaussianos se tiene el software DISPER5.2, que permite evaluar de una manera rápida y sencilla la dispersión de una gran cantidad de contaminantes atmosféricos en el aire como monóxido de carbono (CO), monóxido de nitrógeno (NO), hidrocarburos, cloro, plomo, partículas en suspensión, entre otros [4].

Los modelos eulerianos son aplicables a escalas global, mesoescalar, regional o local. Permiten el procesamiento numérico en paralelo con gran eficiencia y generan información meteorológica en lugares exentos de monitoreo directo [5]. Uno de los modelos eulerianos de última generación es el modelo Weather Research and Forecasting (WRF) de código libre, que se utiliza para pronóstico e investigación meteorológica. Es un modelo numérico de mesoescala no hidrostático de previsión del tiempo y de contaminantes atmosféricos, creado como un sistema operacional flexible y eficiente computacionalmente y ofrece una avanzada descripción física, química y numérica de la atmósfera [6], [7]. Incluye varios núcleos dinámicos para la resolución de las ecuaciones empleadas en la simulación, paquetes de física, programas de inicialización como el WRF-Var para la asimilación de datos y el WRF/ Chem para la simulación de la química de los contaminantes [8].

Para visualizar la información generada por los modelos se emplean herramientas de in- terpolación espacial, que son de utilidad para diversos casos de estudio como por ejemplo el análisis de contaminación acústica [9], contaminación atmosférica, entre otros. Un software de gran utilidad para desarrollar esta actividad es el Sistema de Información Geográfico ArcGIS que emplea el método de ponderación de distancias inversas (IDW, Inverse Distance Weighting [10], [11], que utiliza combinaciones lineales de los datos de los puntos conocidos para estimar los valores de locaciones desconocidas [12].

La correcta implementación de estos modelos permite identificar la contribución de cada una de las fuentes, así como evaluar el impacto que posee cada uno de los contaminantes sobre la calidad de aire en la región estudiada [13].

En este trabajo, se reporta un estudio preliminar de la aplicación de dos modelos de dispersión de los contaminantes atmosféricos. En primera instancia se describe la configuración de los modelos; luego se detalla la modelación y finalmente se comparan los resultados obtenidos entre los modelos con la finalidad de establecer el más adecuado para zonas topográficamente complejas como la ciudad de Cuenca.

\section{MATERIALES Y MÉTODOS}

\subsection{Sitio de estudio}

Para la zona de estudio se consideró la ciudad de Cuenca ubicada en un valle interandino de la sierra sur ecuatoriana, a una altitud promedio de 2550 metros sobre el nivel del mar. Las coordenadas geográficas centrales de la ciudad son $2^{\circ}$ 53'50.79"S y $79^{\circ}$ 0'15.82"W. La superficie del área urbana de la ciudad es de aproximadamente $72 \mathrm{~km}^{2}$.Topográficamente se pueden distinguir tres terrazas, la primera con alturas que van desde los 2560 hasta los $2620 \mathrm{msnm}$, llamada Loma del Cullca; la terraza central donde se emplaza el centro histórico de la ciudad con curvas de nivel entre 2560 y $2520 \mathrm{msnm}$ y la tercera terraza considerada la parte baja, es una planicie que se eleva unos pocos metros sobre los cauces de los ríos y está rodeada por un cordón de colinas bajas y quebradas, las curvas de nivel están por el orden de los 2520 y 2500 msnm.

La Fig. 1 muestra de manera detallada la topografía del sitio de estudio. 
Fig. 1. TOPOGRAFÍA DE LA CIUDAD DE CUENCA

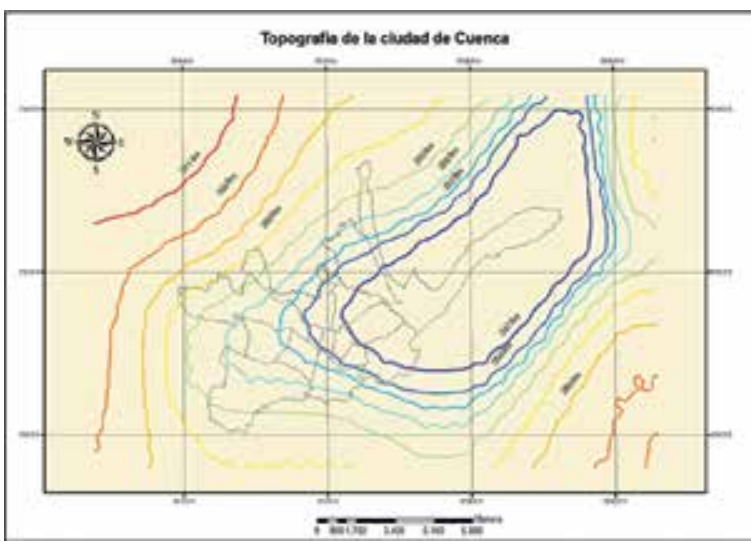

Fuente: autores.

\section{2 Modelos}

Los modelos de dispersión resuelven la siguiente ecuación para estimar la dispersión de los contaminantes emitidos a la atmósfera:

$$
\frac{\partial c}{\partial t}=-c \nabla \cdot \vec{v}-\vec{v} \cdot \nabla c-\nabla \cdot\left(\left\langle c^{\prime} \vec{v}^{\prime}\right\rangle\right)+Q-S
$$

En donde $\mathrm{C}$ es la concentración en $\mathrm{kg} / \mathrm{m}^{3}, \vec{v}$ es el vector velocidad del viento en $\mathrm{m} / \mathrm{s}, \nabla$ es la variación con respecto al espacio (tres dimensiones independientes), $\partial \mathrm{C} / \partial \mathrm{t}$ es la variación local de la concentración promedio de un contaminante respecto al tiempo, $-\vec{v} \cdot \nabla \mathrm{C}$ es la advección de masa por efecto del viento promedio, $\nabla \cdot\left(\left\langle c^{\prime} \vec{v}^{\prime}\right\rangle\right)$ es la divergencia del flujo turbulento del contaminante ( $<>$ denota promedio y ' fluctuaciones en torno al promedio), $\mathrm{Q}$ son las fuentes en $\mathrm{kg} / \mathrm{m}^{3} \mathrm{~s}, \mathrm{~S}$ los sumideros en $\mathrm{kg} / \mathrm{m}^{3} \mathrm{~s}$ [14].

Para la modelación de la dispersión de los contaminantes atmosféricos de la ciudad de Cuenca, se emplearon dos modelos numéricos, DISPER5.2 y Weather Research and Forecasting with Chemistry (WRF/Chem), se empleó también el Sistema de Información Geográfico ArcGIS 9.3 para el postprocesamiento y visualizador de los resultados modelados.

\subsubsection{Modelo DISPER 5.2}

Para la modelación de la dispersión de los contaminantes con el modelo DISPER 5.2 se empleó un mapa de la ciudad en el cual se georeferenciaron los 19 puntos de monitoreo con la ayuda del software ArcGIS 9.3. A continuación fue necesario ingresar para cada punto el número de vehículos por hora que circularon durante la jornada de medición, así como las condiciones meteorológicas como temperatura, velocidad y dirección del viento. Otra variable importante para la modelación fue el parámetro de estabilidad atmosférica de Pasquill (K), que se calculó por medio del ángulo de insolación para cada punto y hora de monitoreo. Para esto se empleó el software on-line Sun EarthTools [15]. Finalmente, con todos los datos de entrada requeridos se calculó la dispersión de los contaminantes [4]. En la Fig. 2 se muestra el diagrama de flujo del modelo DISPER 5.2.

Fig. 2. DIAGRAMA DE FLUJO DEL PROGRAMA DISPER 5.2

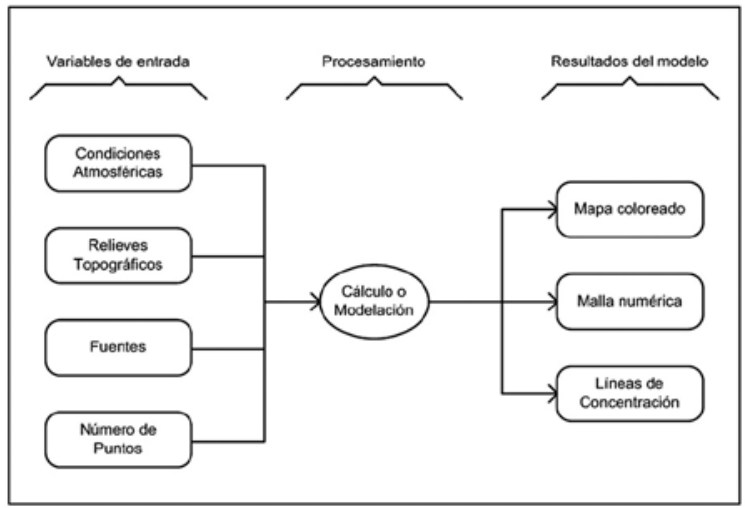

Fuente: autores.

\subsubsection{Modelo WRF/Chem}

Para la obtención de los datos geográficos se partió de información satelital (NCAR Earth System Laboratory (NESL), Mesoscale \& Microscale Meteorology Division) que cuenta con la información geográfica a nivel global e incluye datos de topografía, tipo de suelo, uso de suelo, entre otros [16]. En la Fig. 3 se muestran los dominios configurados para la simulación con el modelo WRF/Chem.

Los datos meteorológicos que se emplearon para la modelación con el programa WRF son datos satelitales de la National Climatic Data Center (NCDC) y National Oceanic and Atmospheric Administration (NOAA). Los datos se reportan cada 6 horas con un pronóstico de hasta 180 horas. Los archivos Global Forecast System (GFS) descargados son de tipo GRIB2, en celdas de $0,5^{\circ} \times 0,5^{\circ}$, es decir, celdas de aproximadamente $55 \mathrm{Km} \times 55 \mathrm{Km}$ [17]. 
Fig. 3. CONFIGURACIÓN DE LOS DOMINIOS PARA LA SIMULACIÓN CON EL WRF/CHEM

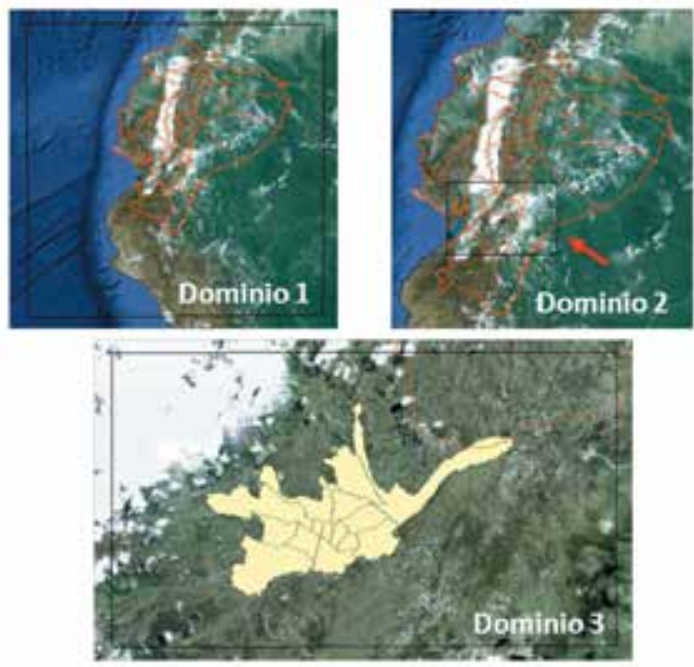

Fuente: autores.

Con la información geográfica y meteorológica se procedió al pre-procesamiento de los datos para la configuración de los dominios y para establecer los datos meteorológicos en el formato requerido por el modelo. Para el pronóstico de la meteorología se inició la simulación desde el día 11 de junio del 2012 a las 06:00 hasta el día 13 de junio del 2012 a las 06:00, es decir, 48 horas de pronóstico.

Como una segunda etapa se procedió a la modelación de los contaminantes atmosféricos emitidos en la zona urbana de la ciudad de Cuenca con el modelo WRF/Chem, por lo que se realizó un monitoreo de gases al aire ambiente en nueve estaciones ubicadas dentro del área de estudio, dicho monitoreo se efectuó el día 11 de junio del 2012 desde las 06:00 hasta las 19:00 con un periodo de medición de una hora en cada estación. Además del monitoreo de los gases se contabilizó el número de vehículos que circularon por cada punto para la estimación de las emisiones generadas en las diferentes estaciones. Los cálculos para la obtención de las emisiones de las fuentes móviles se basan en las siguientes ecuaciones determinadas en el estudio realizado en la Universidad Nacional Autónoma de México en el 2002 [18]:

$$
E_{h k j}=n_{h i j}\left(1-f_{h}\right) F_{j}+n_{h i j} f_{h} F_{j}^{\prime} D_{i k}
$$

Donde $\mathrm{E}_{\mathrm{hkj}}$ es la emisión en el segmento k de la vía i a la hora $\mathrm{h}$ del vehículo tipo $\mathrm{j} \cdot \mathrm{n}_{\mathrm{hij}}$ es el número de vehículos tipo $\mathrm{j}$ en la vía i a la hora $\mathrm{h} \cdot \mathrm{f}_{\mathrm{h}}$ es la fracción de vehículos en frío que circula a la hora $\mathrm{h} \cdot \mathrm{F}_{\mathrm{j}} \mathrm{F}_{\mathrm{j}}^{\prime}$ son los factores de emisión de vehículos tipo $\mathrm{j}$ en caliente y en frío. $\mathrm{D}_{\mathrm{ik}}$ es la longitud del segmento $\mathrm{k}$ de la vía i.

La emisión por celda del dominio de trabajo se calcula de la siguiente manera:

$$
E_{n h}=\sum_{k=1}^{m_{n}} \sum_{j=1}^{\iota} E_{h k j}
$$

Donde $\mathrm{E}_{\mathrm{nh}}$ es la emisión en la celda $\mathrm{n}$ a la hora $\mathrm{h}\left(\mathrm{kg} / \mathrm{km}^{2} . \mathrm{h}\right) . \mathrm{E}_{\mathrm{hkj}}$ es la emisión en el segmento $\mathrm{k}$ de la vía i a la hora h del vehículo tipo j (kg/h). $\mathrm{k}$ es el índice de segmentos en la celda $\mathrm{n} \cdot \mathrm{m}_{\mathrm{n}}$. es el número de segmentos en la celda $\mathrm{n} \cdot \mathrm{j}$ es el tipo de vehículo y l es el número de tipo de vehículos. Como se muestra en 3 se requiere de la fracción de vehículos en frío que circula a la hora h, es decir, los vehículos que inician su trayectoria en el punto de monitoreo. Sin embargo, para el estudio, se consideró la fracción de vehículos en frío como cero y se empleó únicamente el factor de emisión para vehículos en caliente.

Por otra parte, para la obtención de los factores de emisión de los vehículos tanto livianos como pesados, es necesario conocer la velocidad de circulación de los mismos en km/h. En la tabla I se muestran las velocidades promedios de los vehículos en las diferentes estaciones monitoreadas (valores supuestos) y sus respectivos factores de emisión para los contaminantes $\mathrm{CO}$ y óxidos de nitrógeno $\left(\mathrm{NO}_{\mathrm{x}}\right)$. Estos factores se tomaron como referencia del estudio realizado en la Universidad Nacional Autónoma de México [18]:

Con todas estas variables se aplicaron (2) y (3) para el cálculo de las emisiones de los contaminantes $\mathrm{CO}$ y NO generadas por las fuentes móviles en los diferentes puntos de análisis. Además, con las emisiones obtenidas para el contaminante $\mathrm{NO}_{x}$ se procedió a realizar la respectiva especiación química para establecer el porcentaje de emisiones correspondientes al dióxido de nitrógeno $\left(\mathrm{NO}_{2}\right)$ y al $\mathrm{NO}$.

Para la especiación química se consideró que del total de las emisiones de $\mathrm{NO}_{\mathrm{x}}$ el $90 \%$ de estas corresponden al contaminante monóxido de nitrógeno (NO) y el $10 \%$ al dióxido de nitrógeno $\left(\mathrm{NO}_{2}\right)$ [19]. 
En la tabla II se muestran las emisiones estimadas en $\mathrm{mol} / \mathrm{km}^{2}$.h para los contaminantes $\mathrm{CO}$, $\mathrm{NO}$ y $\mathrm{NO}_{2}$, en las diferentes estaciones de monitoreo para la fecha y hora en la que se realizó el estudio.

Con los datos de emisiones listos, se procedió a generar el archivo netCDF necesario para el modelo WRF/Chem, para ello se empleó un software para modelación fotoquímica en el cual se ingresaron las variables requeridas por el modelo. Una vez generado el archivo de emisiones, se ingresó en el modelo WRF/Chem para su ejecución. El diagrama de flujo para la ejecución del modelo WRF/Chem se muestra en la Fig. 4.

TABLA I

FACTORES DE EMISIÓN PARA LOS CONTAMINANTES CO Y NO

\begin{tabular}{|c|c|c|c|c|c|c|c|}
\hline \multirow[t]{2}{*}{ Estación } & \multirow{2}{*}{$\begin{array}{l}\text { Número de vehículos } \\
\text { livianos }\end{array}$} & \multirow{2}{*}{$\begin{array}{l}\text { Número de vehículos } \\
\text { pesados }\end{array}$} & \multirow{2}{*}{$\begin{array}{l}\text { Velocidad } \\
(\mathrm{Km} / \mathrm{h})\end{array}$} & \multicolumn{2}{|c|}{$\begin{array}{l}\text { Factor de emisión vehículos } \\
\text { livianos }(\mathrm{Kg} / \mathrm{Km})\end{array}$} & \multicolumn{2}{|c|}{$\begin{array}{c}\text { Factor de emisión vehículos } \\
\text { pesados }(\mathrm{Kg} / \mathrm{Km})\end{array}$} \\
\hline & & & & $\mathrm{CO}$ & $\mathrm{NO}_{\mathrm{x}}$ & $\mathrm{CO}$ & $\mathrm{NO}_{\mathrm{x}}$ \\
\hline BALZAY & 895 & 133 & 40 & 50 & 2,5 & 10 & 17,5 \\
\hline TEC & 887 & 60 & 20 & 80 & 1,5 & 20 & 22,5 \\
\hline BAS & 1507 & 113 & 50 & 45 & 2,9 & 7 & 16 \\
\hline BCB & 753 & 58 & 20 & 80 & 1,5 & 20 & 22,5 \\
\hline VEG & 364 & 53 & 30 & 60 & 2 & 15 & 19 \\
\hline HDM & 81 & 21 & 40 & 50 & 2,5 & 10 & 17,5 \\
\hline CAI & 481 & 69 & 40 & 50 & 2,5 & 10 & 17,5 \\
\hline UPS & 734 & 106 & 30 & 60 & 2 & 15 & 19 \\
\hline CRB & 448 & 50 & 50 & 45 & 2,9 & 7 & 16 \\
\hline
\end{tabular}

TABLA II

EMISIONES ESTIMADAS DE CO, NO Y NO 2 PARA EL 11 DE JUNIO DEL 2012

\begin{tabular}{|c|c|c|c|c|}
\hline Estación & Hora (hh:mm) & Emisiones $\mathrm{CO}\left(\mathrm{mol} / \mathrm{km}^{2} \mathrm{~h}\right)$ & Emisiones $\mathrm{NO}\left(\mathrm{mol} / \mathrm{km}^{2} \mathrm{~h}\right)$ & Emisiones $\mathrm{NO}_{2}\left(\mathrm{~mol} / \mathrm{km}^{2} \mathrm{~h}\right)$ \\
\hline BALZAY & $06: 38-07: 38$ & 3176228,571 & 171803,775 & 19153,152 \\
\hline TEC & 08:02 - 09:02 & 1907085,714 & 38679,615 & 4312,109 \\
\hline BAS & 09:18 - 10:18 & 1494630,714 & 73490,879 & 8192,963 \\
\hline $\mathrm{BCB}$ & $10: 45-11: 45$ & 1929714,286 & 41776,020 & 4657,304 \\
\hline VEG & $12: 51-13: 51$ & 711385,714 & 29772,600 & 3319,130 \\
\hline HDM & $14: 14-15: 14$ & 152142,857 & 11115,000 & 1239,130 \\
\hline CAI & $15: 26-16: 26$ & 477128,571 & 25377,300 & 2829,130 \\
\hline UPS & $16: 38-17: 38$ & 1727421,429 & 71972,940 & 8023,739 \\
\hline CRB & 18:06 - 19:06 & 380900,000 & 21285,888 & 2373,009 \\
\hline
\end{tabular}


Fig. 4. DIAGRAMA DE FLUJO DEL SISTEMA DE MODELACIÓN WRF/CHEM

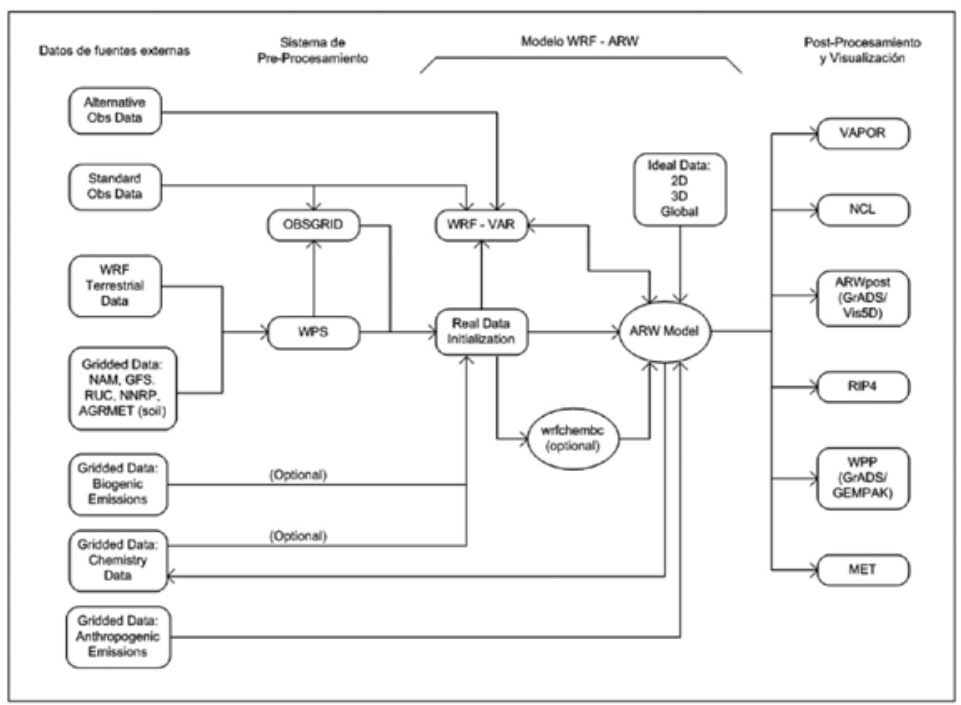

Fuente: Peckham et al. [20].

\subsubsection{Sistema de Información Geográfica ArcGIS 9.3}

Para el posprocesamiento de los resultados de la simulación, tanto de la parte meteorológica como química, se empleó el Sistema de Información Geográfico ArcGIS 9.3 para la visualización de los mapas meteorológicos y de dispersión de los contaminantes atmosféricos estudiados, empleando para ello la herramienta de análisis espacial IDW que resuelve la siguiente ecuación [21], [22]:

$$
\hat{Z}\left(S_{0}\right)=\sum_{i=1}^{n} \lambda_{i} Z\left(S_{i}\right) i=1, \ldots, n
$$

Donde $\hat{Z}\left(\mathrm{~S}_{0}\right)$ es el valor estimado en el punto interpolado $\mathrm{S}_{0} ; \mathrm{n}$ es el número de observaciones vecinas usadas para la estimación y $\lambda_{i}$ es el peso dado al valor observado $Z\left(S_{i}\right)$ en las cercanías del valor $S_{0}[23]$. Los pesos $(\lambda)$ de los valores son determinados con el fin de asegurar que el error promedio para el modelo sea cero y además la varianza del error es minimizada [22], lo cual entrega una predicción no sesgada [11].

\section{RESULTADOS Y DISCUSIÓN}

\section{Modelo DISPER 5.2}

En la Fig. 5, se muestra la modelación obtenida con el modelo DISPER5.2 para el monóxido de carbono para el día 11 de junio del 2012. Al realizar la modelación del contaminante $\mathrm{CO}$ en los diferentes puntos de monitoreo, se presentó una mayor concentración en el centro de la ciudad, proveniente del punto ubicado en la Estación de Servicio de la Av. Solano con una dirección del viento hacia el Nornordeste.

Fig. 5. MODELACIÓN CON EL MODELO DISPER 5.2 PARA EL MONÓXIDO DE CARBONO (CO)

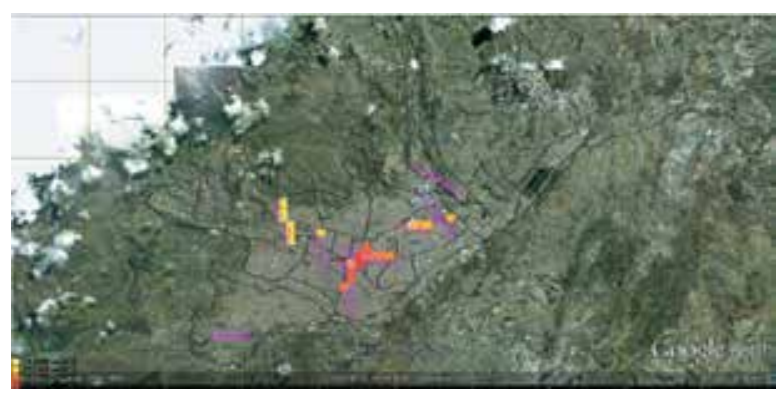

Fuente: autores.

\section{Modelo WRF/Chem}

\section{Pronóstico de la meteorología}

En la Fig. 6 se indican los pronósticos de temperatura obtenidos con el WRF para el dominio 1 correspondiente a Ecuador, en donde se aprecia que la zona costera y oriental del país presentan las temperaturas más elevadas, mientras que en la zona de la Cordillera de los Andes se registran valores por debajo de los $20^{\circ} \mathrm{C}$. 
Fig. 6. PRONÓSTICO DE TEMPERATURA PARA EL ECUADOR OBTENIDOS CON EL MODELO WRF; A) 07:00 DEL 11/06/2012; B) 05:00 DEL 12/06/2012
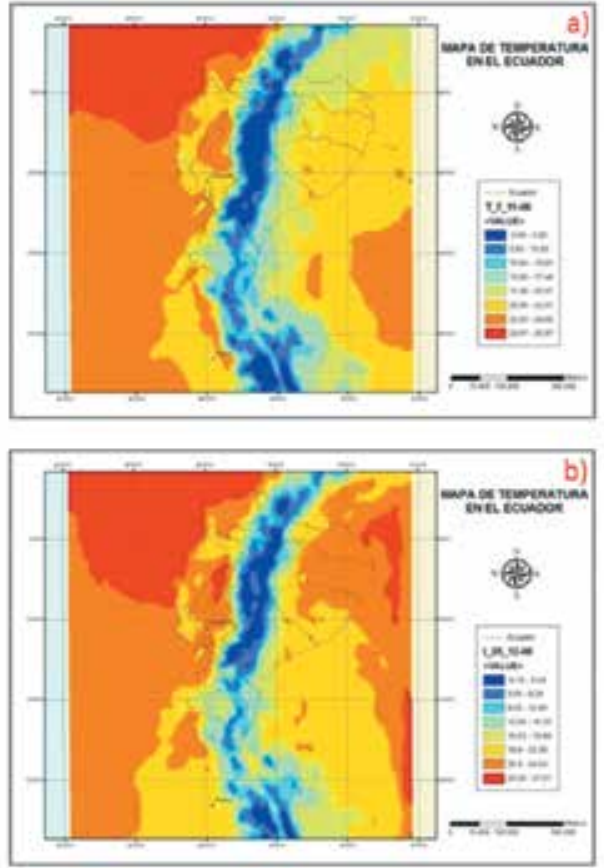

Fuente: autores.

Fig. 7. PRONÓSTICO DE TEMPERATURA PARA CUENCA OBTENIDOS CON EL MODELO WRF; A) 23:00 DEL 11/06/2012; B) 05:00 DEL $13 / 06 / 2012$
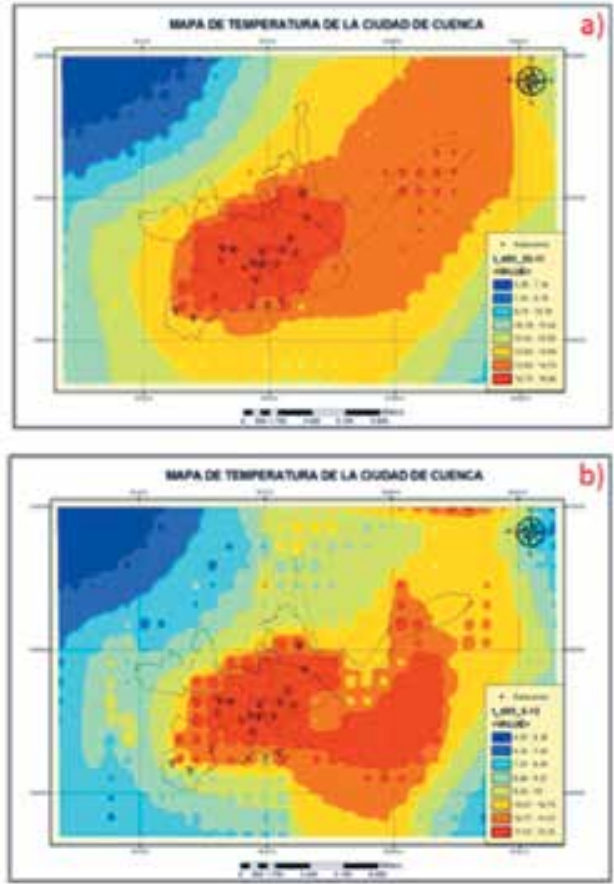

Fuente: autores.
Las mayores temperaturas obtenidas en la ciudad de Cuenca (dominio 3) se concentran en la parte central del dominio. Se observa también que en la parte superior izquierda de la malla se dan las temperaturas más bajas, dado que en esta zona se encuentra ubicado el Parque Nacional El Cajas (Fig. 7).

\section{Modelación de la dispersión de los contaminantes atmosféricos}

En las figs. 8 a 10 se presentan algunos de los mapas obtenidos de la modelación con el WRF/ Chem realizada para el día 11 de junio de 2012.

Como se puede observar en la Fig. 8 las mayores concentraciones de monóxido de carbono se registran en horarios de 09:00 - 10:00 y de 12:00 - 13:00 con valores superiores a $33000 \mu \mathrm{g} / \mathrm{m}^{3}$, pudiendo deberse al elevado flujo vehicular registrado en estos periodos. Se observa también que la dispersión del contaminante durante el día se dirige hacia el Suroeste de la ciudad a causa de los vientos predominantes.

En la Fig. 9 se muestran los resultados obtenidos de la modelación del contaminante dióxido de nitrógeno. Como se puede observar las mayores concentraciones se dan en el horario de 09:00 a 10:00, con valores máximos de $1668.95 \mu \mathrm{g} / \mathrm{m}^{3}$. Además, se puede apreciar que la dispersión del $\mathrm{NO}_{2}$ a lo largo del día se dirige hacia el Suroeste de la ciudad, pudiendo deberse a la dirección de los vientos. Las menores concentraciones obtenidas en la simulación pueden atribuirse a transformaciones químicas del contaminante por acción de la radiación solar.

Con respecto a la simulación obtenida para el Ozono $\left(\mathrm{O}_{3}\right)$, en la Fig.10 se observa que las mayores concentraciones se registran en las periferias de la ciudad, con valores superiores a los $90 \mu \mathrm{g} /$ $\mathrm{m}^{3}$, mientras que las mínimas se dan dentro de la zona urbana de Cuenca. El Ozono es un contaminante secundario que se forma por reacciones fotoquímicas de contaminantes primarios como el $\mathrm{NOx}\left(\mathrm{NO}_{2}, \mathrm{NO}\right)$, por lo que resulta lógica la modelación obtenida dado que para el contaminante $\mathrm{NO}_{2}$ se obtuvieron concentraciones mínimas en las periferias; es decir, que una vez emitido a la atmósfera, parte de este contaminante se transforma en ozono por acción de la radiación solar.

De acuerdo con los resultados obtenidos con los diferentes programas computacionales utiliza- 
Fig. 8. DISPERSIÓN DEL CO EN MG/M3, OBTENIDOS CON EL WRF/CHEM; A) 09:00 - 10:00; B) 12:00 - 13:00
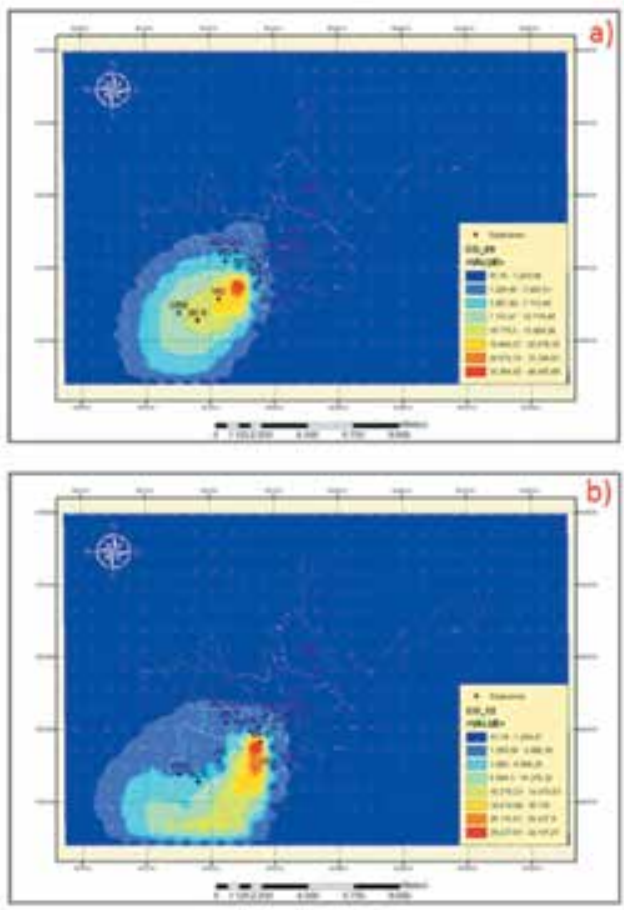

Fuente: autores.

Fig. 9. DISPERSIÓN DEL NO ${ }_{2}$ EN MG/M ${ }^{3}$, OBTENIDOS CON EL WRF/CHEM; A) 09:00 - 10:00; B) 14:00 - 15:00
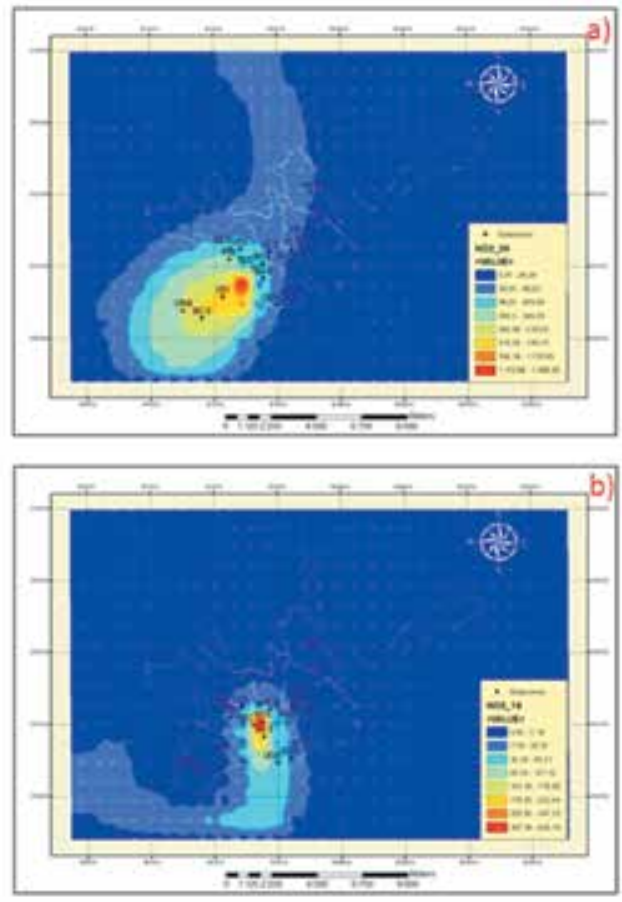

Fuente: autores. dos en este estudio, se encontraron algunas limitaciones que se detallan a continuación:

La dispersión obtenida con el modelo DISPER5.2 no permite visualizar correctamente la escala de colores, así como los valores de concentración representados en la parte inferior izquierda del mapa. La ventana de trabajo del modelo no permite emplear mapas de gran escala, ya que no existe la opción de desplazarse a través de toda la imagen, por lo que el área de estudio es incompleta y no se obtiene una buena resolución. El modelo DISPER5.2 realiza la modelación de manera puntual, es decir, que al ingresar los datos de entrada no los relaciona para generar la dispersión del contaminante.

Para este estudio en particular se presentó una limitante en el modelo WRF/Chem al no disponer de suficiente información para alimentar al mismo, por lo que no se obtuvieron los resultados esperados de pronóstico para la contaminación atmosférica. Sin embargo, se escogió este modelo por ser el que menos limitaciones presenta y es uno de los más recientes modelos numéricos que simulan al mismo tiempo la meteorología y los procesos de transporte químico de los contaminantes en la atmósfera; lo que le proporciona una ventaja conceptual y pragmática con relación a otros enfoques que tratan con modelos separados tanto para la meteorología como el transporte químico.

\section{Validación del Modelo WRF/CHEM}

Para la validación se realizaron mediciones en 9 puntos de la ciudad de Cuenca con un equipo de mediciones de gases como monóxido de carbono, dióxido de nitrógeno, monóxido de nitrógeno marca TESTO. Los resultados se muestran en la Fig. 11.

Al aplicar el análisis estadístico mediante el software SPSS y GraphPadPrism 5 por medio del uso del estadígrafo de Mann-Whitney se obtuvo para el contaminante $\mathrm{CO}$ un valor de $\mathrm{P}>0.05$ (0.8633), lo que significa que no existe una diferencia significativa entre los datos reales y los datos modelados, por lo tanto la modelación con el programa WRF/Chem es buena. Sin embargo, para el contaminante $\mathrm{NO}_{2}$ el valor de $\mathrm{P}$ es menor a 0.05 (0.04) que implica una diferencia significativa entre los datos, es decir, la relación entre estos es débil, lo cual puede ser debido a las reacciones químicas de los contaminantes en la atmósfera que no son consideradas por el equipo al momen- 
Fig. 10. DISPERSIÓN DE 03 EN MG/M3, OBTENIDOS CON EL WRF/CHEM; A) 09:00 - 10:00; B) 12:00 - 13:00
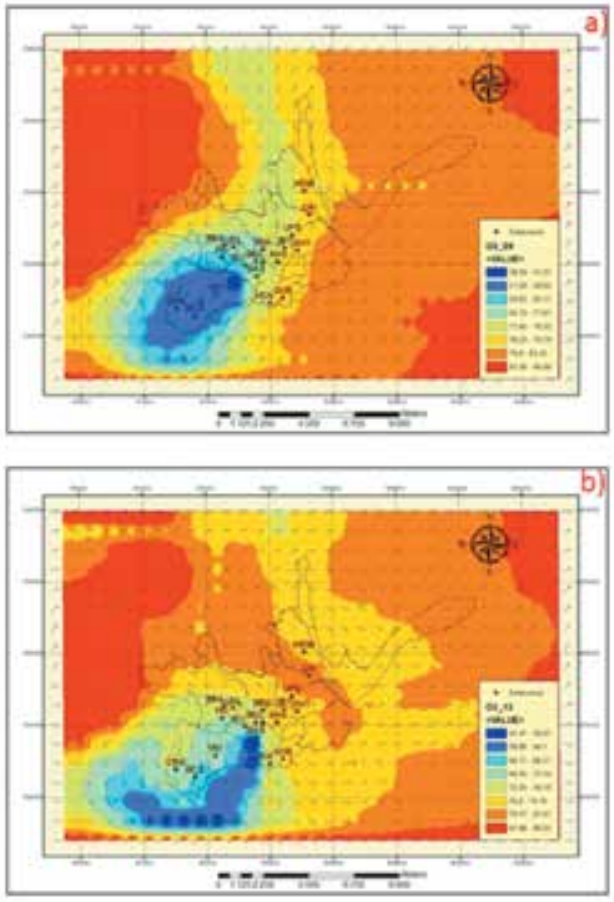

Fuente:autores.

Fig. 11. VALIDACIÓN DEL MODELO WRF/CHEM; A) MONÓXIDO DE CARBONO; B) DIÓXIDO DE NITRÓGENO
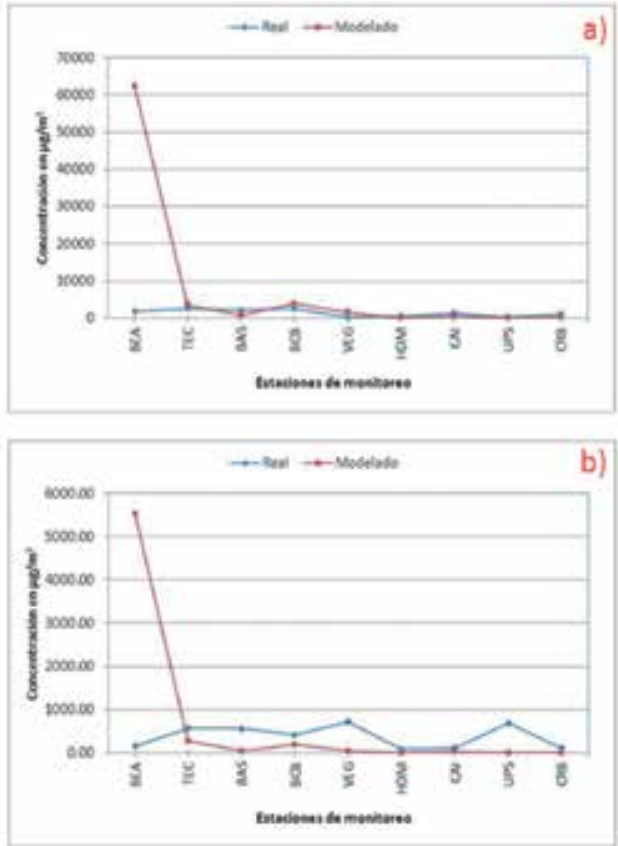

Fuente: autores. to del monitoreo, mientras que, para la simulación con el WRF/Chem se integran todos estos aspectos junto con las condiciones de meteorología para el respectivo pronóstico.

\section{CONCLUSIONES}

El software Canarina tiene una serie de limitantes por lo cual no pudo ser utilizado para esta investigación, es como un software didáctico que no tiene mucha aplicación en esta.

Se ha seleccionado el modelo WRF/Chem para realizar la modelación de los contaminantes atmosféricos de la ciudad de Cuenca debido a que presenta la ventaja de integrar la meteorología y los procesos de transporte químico de los contaminantes en la atmósfera; y como un instrumento para generar y visualizar los mapas resultantes de la modelación se empleó el Sistema de Información Geográfico ArcGIS, ya que es un programa muy útil para estudios geoestadísticos por la diversidad de herramientas del que dispone.

Se debe evaluar con mayor integralidad el desempeño del modelo WRF/Chemy para afinar aquellos elementos que pueden estar contribuyendo con las mayores incertidumbres en los resultados del modelo.

Para el pronóstico de la parte química se necesita de mayor información de la contaminación de la ciudad de Cuenca, la cual permita generar un historial de emisiones, con datos, horarios de los diferentes contaminantes para alimentarlos al modelo WRF/Chem.

Se debe estudiar más a profundidad las herramientas disponibles en el modelo para la parte meteorológica y así poder emplear como datos de entrada las condiciones meteorológicas locales y conocer el grado de aproximación con los datos satelitales que se utilizaron en el presente estudio.

\section{REFERENCIAS}

[1] USEPA, “Technology transfer network. Support center for regulatory atmospheric modeling. Air quality models", United States Environmental Protection Agency, 12, Dic., 2011. [Online]. Disponible: http://www.epa. gov/ttn/scram/aqmindex.htm.

[2] C. Bustos, "Aplicación de modelos de dispersión atmosférica en la evaluación de impacto ambiental: Análisis del proceso", (Tesis de Maestría, UCH), Santiago de Chile, Chile, 2004. 
[3] A. Misra, M. Roorda, H. Maclean, "An integrated modelling approach to estimate urban traffic emissions", Atmos. Environ., vol. 73, pp. 81-91, Jul. 2013.

[4] Canarina Algoritmos Numéricos, "Aplicación informática para la simulación por ordenador de la contaminación atmosférica", España, 2001, pp. 26-27.

[5] R. Parra, "Evaluación preliminar de la temperatura media en superficie del Ecuador para el año 2010, obtenida mediante el modelo Weather Research Forecasting (WRF)", Avances, vol. 4, no 2, pp. C27-C35, Dic. 2012.

[6] M. Ritter, M. Müller, T. Ming-Yi, E. Parlow, "Air pollution modeling over very complex terrain: An evaluation of WRF-Chem over Switzerland for two 1-year periods", Atmos. Res., pp. 132-133, 209-222, Oct.-Nov. 2013.

[7] J. Rivera, O. Sánchez, E. Concepción, "Metodología para el diseño de una red de monitoreo para la calidad del aire. Aplicación para la ciudad de Piura", RPGA, vol. 3, pp. 95-103, 2011.

[8] W. Wang, C. Bruyère, M. Duda, J. Dudhia, D. Gill, H-C. Lin, J. Michalakes, S. Rizvi, X. Zhang, "Advanced Research WRF (ARW)" Version 3 Modeling User's, Guide, Mesoscale \& Microscale, Meteorology Division, National Center for Atmospheric Research (NCAR)", USA, 2011, pp. 8-1, 8-4.

[9] V. Vázquez, A. Astudillo, C. Espinoza, “Elaboración de un mapa acústico empleando un Sistema de Información Geográfica para la zona urbana de Cuenca", ClYTA, vol. 1, pp. 1-9, Ene. 2011.

[10] K. Johnston, J. VerHoef, K. Krivoruchko, N. Lucas, Using ArcGis Geostatistical Analyst.ESRI, USA, 2001.

[11] M. Villatoro, C. Henríquez, F. Sancho, “Comparación de los interpoladores IDW y Kriging en la variación espacial de PH, CA, CICE y P del Suelo", Agronomía Costarricense, vol. 32, no 1, pp. 95-105, Mar. 2008.

[12] E. Roberts, R. Sheley, R. Lawrence, "Using sampling and Inverse Distance Weighted Modeling for mapping invasive plants", West N Am Naturalist, vol. 64, no 3, pp. 312-323, Ago. 2004.

[13] R. Fernández, D. Allende, F. Castro, P. Cremades, E. Puliafito, "Modelado regional de la calidad de aire utilizando el Modelo WRF/Chem: Implementación de datos globales y locales para Mendoza". AVERMA, vol. 14, pp. 01.43-01.50, 2010.
[14] [14] L. Gallardo, "Modelos de dispersión de contaminantes atmosféricos", CONAMA, Santiago de Chile, Chile, 1997.

[15] SunEarthTools.com, "Outilspour les consommateurs et les concepteurs de l'énergiesolaire", 23-Jul-2012. [Online]. Disponible: http://www.sunearthtools.com.

[16] NCAR Earth System Laboratory (NESL), Mesoscale \& Microscale Meteorology Division, "Base de datos geográficos para modelación", National Center for Atmospheric Research, 15 Jun. 2011. [Online]. Disponible: http://www.mmm.ucar.edu/.

[17] NCDC, NOAA, "Base de datos meteorológicos de pronóstico", National Climatic Data Center, National Oceanic and Atmospheric Administration, 11 Ene. 2012. [Online]. Disponible: http://www.ncdc.noaa. gov/oa/ncdc.html.

[18] R. García, "Evaluación de escenarios utilizando el Modelo Regional de Calidad del Aire Multiscale Climate Chemistry Model", (Tesis de Doctorado), Centro de Ciencias de la Atmósfera, UNAM, México, 2002.

[19] Radian International, "Manuales del Programa de Inventarios de Emisiones de México", Sacramento, CA, 2000.

[20] S. Peckham, G. Grell, S. McKeen, J. Fast, W. Gustafson, S. Ghan, R. Zaveri, R. Easter, J. Barnard, E. Chapman, C. Wiedinmyer, R. Schmitz, M. Salzmann, S. Freitas, WRF/ Chem Version 3.3 User's Guide, USA, 2011, pp. 6.

[21] C. Gotway, R. Ferguson, G. Hergert, T. Peterson, "Comparison of kriging and inverse distance methods for mapping soil parameters". SOIL SCI SOC AM J, vol. 60, pp. 1237-1247, Jul. 1996.

[22] C. Schloeder, N. Zimmerman, M. Jacobs, "Comparison of methods for interpolating soil properties using limited data", SOIL SCI SOC AM J, vol. 65, pp. 470-479, Mar. 2001.

[23] Z. Lozano, C. Bravo, F. Ovalles, R. Hernández, B. Moreno, L. Piñango, J. Villanueva, "Selección de un diseño de muestreo en parcelas experimentales a partir del estudio de la variabilidad espacial de los suelos", BIOAGRO, vol. 16, no 1, pp. 1-17, Ene. 2004. 